\title{
Integrated approach to designing growth factor delivery systems
}

\author{
Ruth R. Chen, ${ }^{*}{ }^{\dagger}$ Eduardo A. Silva, ${ }^{\dagger}$ William W. Yuen, ${ }^{\dagger}$ Andrea A. Brock, ${ }^{\dagger}$ \\ Claudia Fischbach, ${ }^{\dagger}$ Angela S. Lin, ${ }^{\S}$ Robert E. Guldberg, ${ }^{\S}$ and David J. Mooney ${ }^{\dagger, 1}$ \\ *Department of Biomedical Engineering, University of Michigan, Ann Arbor, Michigan, USA; \\ ${ }^{\dagger}$ Division of Engineering and Applied Sciences, Harvard University, Cambridge, Massachusetts, USA; \\ ${ }^{\ddagger}$ Department of Biomedical Engineering, Saint Louis University, St. Louis, Missouri, USA; and \\ ${ }^{\S}$ School of Mechanical Engineering, Georgia Institute of Technology, Atlanta, Georgia, USA
}

ABSTRACT Growth factors have been widely used in strategies to regenerate and repair diseased tissues, but current therapies that go directly from bench to bedside have had limited clinical success. We hypothesize that engineering successful therapies with recombinant proteins will often require specific quantitative information of the spatiotemporal role of the factors and the development of sophisticated delivery approaches that provide appropriate tissue exposures. This hypothesis was tested in the context of therapeutic angiogenesis. An in vitro model of angiogenesis was adapted to quantify the role of the concentration/gradient of vascular endothelial growth factor [VEGF(165)] on microvascular endothelial cells, and a delivery system was then designed, based on a mathematical model, to provide the desired profile in ischemic mice hindlimbs. This system significantly enhanced blood vessel formation, and perfusion and recovery from severe ischemia. This general approach may be broadly applicable to growth factor therapies. Chen, R. R., Silva, E. A., Yuen, W. W., Brock, A. A., Fischbach, C., Lin, A. S., Guldberg, R. E., Mooney, D. J. Integrated approach to designing growth factor delivery systems. FASEB J. 21, 3896-3903 (2007)

Key Words: tissue engineering • in vitro models • angiogenesis

Current molecular-Level understanding of processes regulating tissue formation (e.g., angiogenesis) suggests recombinant growth factors may provide disease therapies, but clinical efforts that go directly from discovery to clinical application have often been unsuccessful $(1,2)$. The success of therapeutic growth factor delivery strategies may depend on the ability to intelligently design delivery systems based on quantitative delivery parameters.

Tissue development and regeneration depend on tightly coordinated spatial and temporal growth factor signals, and recombinant growth factors have been widely proposed for therapeutic use in the regeneration and repair of diseased tissues (3). Vascular endothelial growth factor (VEGF) is a potent initiator of new vascular network growth, termed angiogenesis (4), and has been the centerpiece of many strategies to treat ischemic diseases (1). However, delivery strategies thus far have yielded limited success in creating stable, functional vasculature capable of relieving local ischemia (5), and this likely relates both to the rapidly depleted, poorly controlled local concentrations of VEGF that result from the typical delivery route (2) and the disorganized, poorly functional vessel networks formed. Furthermore, although the absolute concentration and gradient of VEGF in vitro clearly regulate endothelial cell phenotype and nascent angiogenesis, there is little quantitative information regarding the specific physiologically active concentration range and gradient. Successful therapies using VEGF will likely require knowledge of the cell exposure necessary to elicit a controlled angiogenic response and the design of a VEGF delivery system that can provide therapeutically effective growth factor profiles based on these criteria.

Using a combination of quantitative in vitro methods, we developed an in vitro model of angiogenesis to allow the quantification of VEGF binding on human dermal microvascular endothelial cells (MECs), used this in vitro model to determine the concentration and gradient of VEGF presentation necessary to direct angiogenesis, and then designed a system capable of delivering VEGF in vivo in the desired profile. Based on in vitro evaluation of growth factor controlled angiogenesis, in vivo delivery of growth factors was optimized to enhance recovery from severe ischemia. The approach we describe may also lead to enhanced tissue integration and function when applied to the regeneration of other tissues such as bone or nerves.

\section{MATERIALS AND METHODS}

Cell culture

Dermal MECs were purchased from Cambrex (East Rutherford, NJ, USA) and cultured to confluence at $37^{\circ} \mathrm{C}$ and $5 \%$

\footnotetext{
${ }^{1}$ Correspondence: Harvard University, 319 Pierce Hall, Cambridge, MA 02138, USA. E-mail: mooneyd@deas. harvard.edu doi: 10.1096/fj.06-7873com
} 
$\mathrm{CO}_{2}$ in EGM-2MV containing all supplements. MECs at P4 were used for all experiments.

\section{Bead sprouting assay}

This sprouting assay was adapted from previously described methods (6). Briefly, swollen and sterilized dextran microcarriers (Amersham Biosciences, Piscataway, NJ, USA) were washed in EGM-2MV and combined with MECs in a spinner flask at a seeding density of 5000 cells $/ \mathrm{cm}^{2}$. The flask was alternated between stirring and nonstirring every $30 \mathrm{~min}$ and incubated at $37^{\circ} \mathrm{C}$ and $5 \% \mathrm{CO}_{2}$ to promote even cell attachment $(6,7)$. After $4 \mathrm{~h}$, the beads and cells were left stirring and incubated for $20 \mathrm{~h}$. The cell-coated beads were then collected and incubated in flasks on a shaker for 1-2 days until cells became confluent on the beads. A fibrinogen-bead solution ( $1 \mathrm{ml}$ of cell-coated beads in media, $27 \% \mathrm{w} / \mathrm{v}$ fibrinogen, and $0.5 \% \mathrm{w} / \mathrm{v}$ aprotinin) was then mixed with a thrombin solution $(8.3 \mathrm{U} / \mathrm{ml}$ thrombin) in each well at a ratio of 5:4 fibrinogen-bead solution:thrombin solution. Gels were allowed to set in the incubator for $20 \mathrm{~min}$ before addition of basic EGM-2MV supplemented with growth factors of interest at specific concentrations. Media were changed every $24 \mathrm{~h}$, and gels were maintained for up to 7 days. Gels were rinsed with PBS and fixed with $4 \%$ paraformaldehyde before imaging for sprout quantification.

\section{Sprout imaging}

High resolution images of beads were captured at $\times 100$ and $\times 400$ magnification. Epifluorescence images were captured on the same Olympus microscope. Confocal images were taken using an upright Zeiss LSM510 META microscope (Carl Zeiss Inc., Thornwood, NY, USA). Images were analyzed using IPLab 3.7 (Scanalytics, Rockville, MD, USA). Fluorescence images were background corrected using the rolling ball script (8). Individual cells were segmented by intensity, and the area of segmented sprouting cells was measured. Fluorescence values of sprouts were corrected by subtracting the minimum background intensity value of the image. The number of sprouts per bead and sprout lengths was manually determined, where a sprout is defined as an extension containing more than one cell.

\section{VEGF labeling}

VEGF-A isoform VEGF(165) (Biological Resources Branch of the National Cancer Institute, Bethesda, MD, USA) was used throughout these studies.

Fluorescence labeling of VEGF was achieved by using Alexa Fluor 555 Microscale Protein Labeling Kit (Molecular Probes, Eugene, OR). Labeling was conducted according to instructions provided in the kit. Briefly, a reaction mixture of $50 \mu \mathrm{l}$ of a $1 \mathrm{mg} / \mathrm{ml}$ solution of VEGF, $5 \mu \mathrm{l}$ of $1 \mathrm{M}$ sodium bicarbonate, and an appropriate volume of reactive dye solution calculated based on the protein molecular weight was incubated at room temperature for $15 \mathrm{~min}$. Labeled protein was separated from unreacted dye by centrifugation in gel resin, and labeled protein was collected into $400 \mu \mathrm{l}$ sterile water. The degree of labeling and yield were determined according to equations provided in the kit instructions before use. The bioactivity of labeled protein was confirmed using a proliferation assay with MECs (see Supplemental Fig. 4). Labeled VEGF was used to determine VEGF concentrations in the diffusion chamber experiments by quantification of fluorescence as a function of distance and converting to concentrations using the known VEGF degree of labeling.
The binding assay was performed according to described methods for determining binding on living cells (9). MECs were seeded at 5000 cells $/ \mathrm{cm}^{2}$ in 24 -well plates and grown to $70 \%$ confluence. Cell medium was changed to EGM-2MV without VEGF and incubated overnight. Cells were rinsed twice with PBS and preincubated with EBM-2 media containing a saturating concentration of basic fibroblast growth factor $(300 \mathrm{ng} / \mathrm{ml})$ to saturate nonspecific heparin-sulfate proteoglycans binding sites and $0.1 \%$ BSA for $4 \mathrm{~h}$ at $4^{\circ} \mathrm{C}$ to reduce nonspecific binding to other sites. MECs were then incubated in increasing quantities of ${ }^{125}$ I-VEGF (1-160 ng/ $\mathrm{ml}$ ), and binding was allowed to equilibrate overnight at $4^{\circ} \mathrm{C}$. Cells were washed twice with PBS, lysed with trypsin, and collected. Cells incubated with unlabeled VEGF were collected and counted. Radioactivity of each sample was counted using a gamma counter and normalized to cell number. Nonspecific VEGF binding was determined by incubation with a 200-fold excess of unlabeled VEGF over labeled VEGF (9-12). The number of VEGF molecules bound to MECs was calculated from the raw count and the specific activity of the batch of ${ }^{125}$ I-VEGF used in the assay.

\section{Quantification of VEGF binding using fluorescence resonance transfer technique (FRET)}

MECs were labeled with 5-hexadecanoylaminofluorescein (HEDAF) as the donor fluorophore. One milliliter of HEDAF at a concentration of $10 \mathrm{mg} / \mathrm{ml}$ in DMSO was diluted 10-fold in EGM-2MV, and the media were filtered to remove precipitates. The filtered HEDAF-media were diluted with media to a total volume of $25 \mathrm{ml}$. MECs cultured to confluence in 75 $\mathrm{cm}^{2}$ flasks were incubated with $15 \mathrm{ml}$ of the diluted HEDAFmedia for $3 \mathrm{~h}$ at $37^{\circ} \mathrm{C}$ and $5 \% \mathrm{CO}_{2}$.

Labeled cells were collected and replated at a density of 5000 cells $/ \mathrm{cm}^{2}$ in 96-well tissue culture plates overnight in EGM-2MV without VEGF. The cells were rinsed twice with PBS before incubation with Alexa 555-labeled VEGF for 30 $\min$ at $4^{\circ} \mathrm{C}$ to prevent internalization. VEGF binding was determined by quantifying fluorescence using a Synergy HT Microplate Reader (BioTek Instruments, Winooski, VT, USA) fitted with appropriate wavelength filters. Cells were excited at the excitation wavelength of the donor $(488 \mathrm{~nm})$, and fluorescence was monitored at the emission wavelength of the acceptor $(565 \mathrm{~nm})$. Fluorescence was also determined with unlabeled cells and labeled VEGF to determine autofluorescence, and labeled MECs with labeled BSA to determine nonspecific fluorescence. Autofluorescence and nonspecific fluorescence values were subtracted to determine specific fluorescence resulting from VEGF binding. Fluorescence values were normalized to cell number and then correlated to bound VEGF determined as a function of VEGF concentration from classic radiolabeled binding assays (section on radiolabeled VEGF binding assay) to obtain the actual quantity of VEGF bound by cells in the gels.

\section{Scaffold fabrication}

Mixtures of poly(lactide-coglycolide) (PLG; 85:15, intrinsic viscosity $=0.8 \mathrm{dl} / \mathrm{g}$; and 75:25, intrinsic viscosity $=0.26 \mathrm{dl} / \mathrm{g}$; Alkermes, Cambridge, MA, USA) microspheres (diameter $=5-100 \mu \mathrm{m})$ formed by standard double emulsion were lyophilized with or without VEGF (Biological Resources Branch of the National Cancer Institute) in a solution of MVM alginate, a high-M-containing alginate, (5\% of total polymer mass; ProNova, Oslo, Norway) as described previously (13). Each scaffold layer containing $3 \mathrm{mg}$ of PLG total 
and $50 \mathrm{mg}$ of salt $(250 \mu \mathrm{m}<\mathrm{d}<425 \mu \mathrm{m})$ was mixed separately and then manually compacted on top of each other, and finally compressed to form a single scaffold $(4.7 \mathrm{~mm}$ in diameter, $3 \mathrm{~mm}$ total thickness). Scaffolds were then gasfoamed and particulate-leached as described previously (14, 15). Total protein loaded after fabrication was $1.5 \mu \mathrm{g}$ of VEGF in scaffold layer 1 and $3 \mu \mathrm{g}$ VEGF in scaffold layer 2.

\section{Analysis of VEGF distribution}

Scaffolds were fabricated as described above using Alexa Fluor 488-conjugated BSA (Molecular Probes, Carlsbad, CA, USA) in place of VEGF to examine the spatial distribution of incorporated protein. Scaffolds were imaged using a Leica MZFL III stereodissecting fluorescence microscope (Leica Microsystems, Bannockburn, IL, USA).

\section{Quantification of VEGF release kinetics}

VEGF release kinetics from each layer of the scaffold were determined using ${ }^{125}$ I-labeled recombinant human VEGF (Perkin Elmer, Boston, MA, USA) as a tracer (15). Scaffolds were fabricated as described with the addition of $0.11 \mu \mathrm{Ci}$ radiolabeled VEGF mixed with the bulk quantity of unlabeled VEGF. The total radioactivity of each scaffold layer $(n=4)$ was counted in a gamma counter before incubation at $37^{\circ} \mathrm{C}$ in PBS containing $\mathrm{Ca}^{2+}$ and $\mathrm{Mg}^{2+}$. At specific time points, the radioactivity of the release solution was measured using a WIZARD Automatic Gamma Counter (Perkin Elmer). Cumulative VEGF released from the scaffold at each time point was calculated as a percentage of total protein incorporated.

\section{Mathematical modeling}

The mathematical model described the VEGF profile as a result of diffusion, release from the scaffold, and degradation. The governing equation of the VEGF concentration, $c$, in each of the two layers of the scaffold was

$$
\frac{\partial c_{i}}{\partial t}=D_{V} \nabla^{2} c_{i}+k_{r} c_{o i}-k_{d} c_{i} ; \quad i=1,2
$$

where the index $i$ refers to the layer. The diffusion coefficient of VEGF, $D_{V}=7.0 \times 10^{-7} \mathrm{~cm}^{2} / \mathrm{s}$, was determined by fitting experimental data on VEGF diffusion. VEGF generation was expressed as the product of the release rate, $k_{r}$, and the initial concentration in the layer at implantation, $c_{o i}$. VEGF release was approximated to have two linear regions: a quick release in the first 2 days and a slower subsequent release based profiles of VEGF release from this scaffold system $\left(k_{r}=2.3 \times 10^{-6} \mathrm{~s}^{-1}\right.$ during the first 2 days and $4.7 \times 10^{-7} \mathrm{~s}^{-1}$ thereafter). The VEGF degradation rate $\left(k_{d}=2.31 \times 10^{-4} \mathrm{~s}^{-1}\right)$ was determined experimentally from an in vitro bioactivity assay. The steady-state system was solved in COMSOL Multiphysics using the 2-D axial symmetric diffusion analysis tool with a direct UMFPACK method.

\section{Mouse model of hindlimb ischemia and scaffold implantation}

Layered scaffolds were implanted in 7- to 8-wk-old SCID mice (Taconic, Hudson, NY, USA) that had undergone unilateral ligation of hindlimb blood vessels to create a severe model of hindlimb ischemia (16). Briefly, animals were anesthetized by intraperitoneal injection of a ketamine and xylazine cocktail. The entire hindlimb was shaved and sterilized before an incision was made through the dermis. The external iliac artery and vein and the femoral artery and vein were ligated using 5-0 Ethilon (Ethicon, Somerville, NJ, USA). The vessels were severed between the ligation points, and a layered polymer scaffold was implanted with an orientation such that layer 1 was directly over the sites of ligation and layer 2 extended into the muscles of the inner thigh.

\section{Laser Doppler perfusion imaging (LDPI)}

Measurements of the ischemic/normal limb blood flow ratio were performed on anesthetized animals $(n=5)$ using a LDPI analyzer (Perimed AB, Stockholm, Sweden). Perfusion measurements were obtained by scanning entire hindlimbs. To minimize variability due to ambient light and temperature, the index was expressed as the ratio of ischemic to nonischemic limb blood flow.

\section{Micro-computed tomography (CT) imaging and analysis}

After euthanization, the vasculature of animals was first flushed with warm $0.9 \%$ heparinized saline $(100 \mathrm{U} / \mathrm{ml})$ and then pressure fixed by flushing with $10 \%$ formalin through the left ventricle. Formalin was cleared using heparinized saline just before injection of a 9:1 mixture of the catalyst and contrast compound (Microfil MV-122, Flow Tech, Carver, MA, USA). Specimens were stored at $4^{\circ} \mathrm{C}$ in a humid chamber overnight to allow the contrast agent to polymerize. Dissected hindlimbs were fixed and then decalcified in a formic acid solution (Cal-Ex II, Fisher Scientific, Pittsburgh, PA, USA). Samples were rinsed and stored in $10 \%$ formation at $4^{\circ} \mathrm{C}$ until imaging.

Hindlimb samples were imaged at high resolution $(\mu \mathrm{CT}$ 40; Scanco Medical, Bassersdorf, Switzerland) with the scanner set to a voltage of $55 \mathrm{kVp}$ and a current of $145 \mu \mathrm{A}$. Specimens were scanned at a voxel size of $30 \mu \mathrm{m}$, and a threshold of 100 was applied based on visual interpretation of thresholded 2-D tomograms.

\section{Statistical analysis}

Mean values are shown with error bars indicating the SD, unless indicated otherwise. Statistical analysis was performed by Student's $t$ test, where $P<0.05$ denotes statistical significance.

\section{RESULTS}

To determine the quantitative relation between VEGF (165) presentation and angiogenesis, an in vitro 3-D model (6) was used to quantify the relation between the level and direction of sprouting, the initial stage in angiogenesis, and VEGF concentration and gradient. MECs on beads embedded in a fibrin gel were induced to undergo angiogenic sprouting in response to VEGF in the media (Fig. 1a) at VEGF concentrations that elicited a sprouting response, and sprouting was directly proportional to the concentration of VEGF (Fig. 1b). The concentrations of VEGF noted in this study to significantly effect sprouting are within the same range as previously reported $(6,7,17)$. To quantify the relation between VEGF gradients and sprouting, the sprouting assay was performed in a diffusion chamber that created specific gradients of VEGF across the gel (see Supplemental Fig. 1). In general, higher 

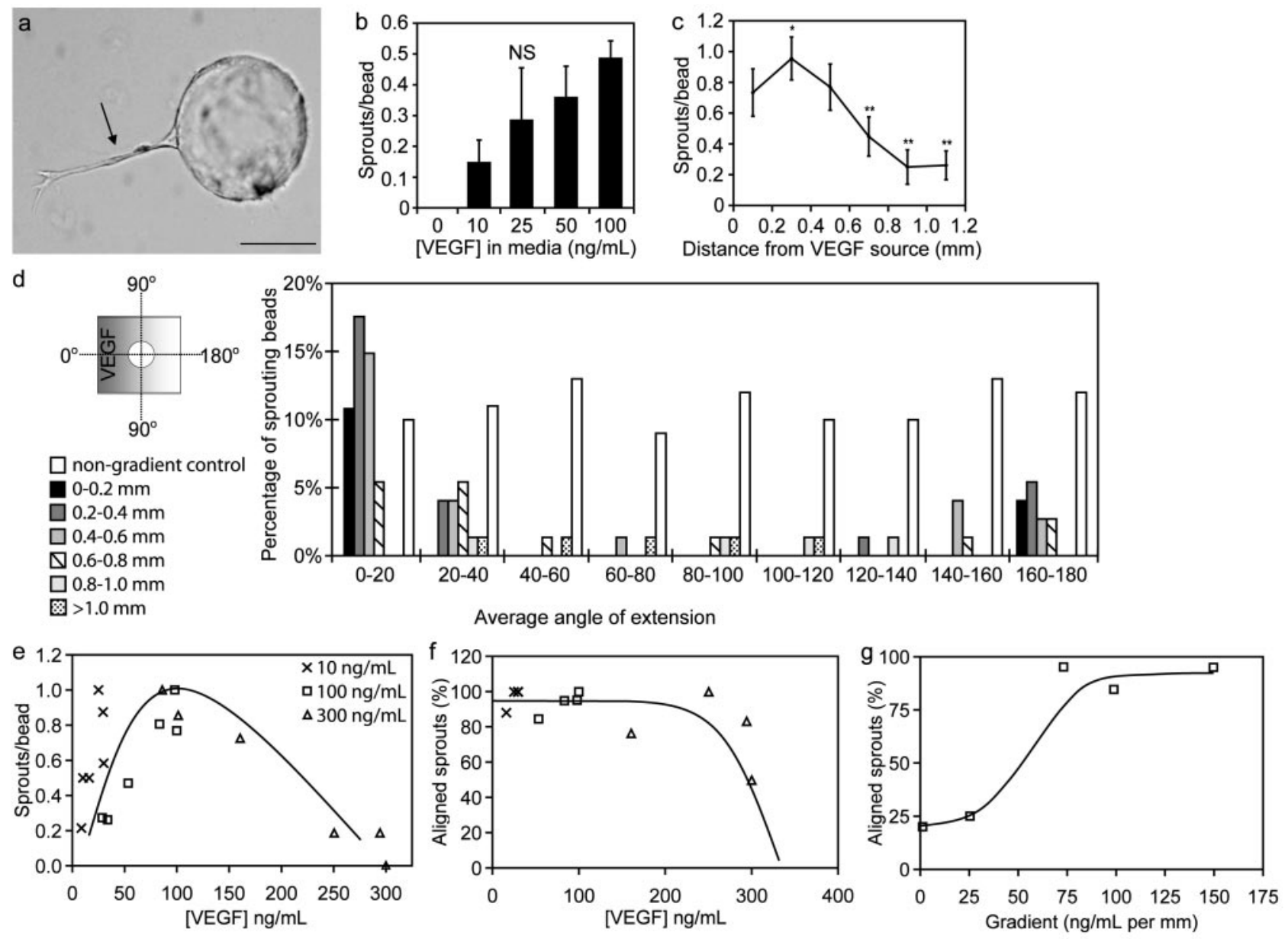

Figure 1. Endothelial cell sprouting in response to VEGF. a) Representative image of a bead with a MEC sprout (arrow). Sprout consists of $>1$ connected MECs that have migrated from bead into fibrin gel. Scale bar $=100 \mu \mathrm{m}$. $b$ ) MECs were seeded on microcarriers embedded in fibrin gel and cultured with $0-100 \mathrm{ng} / \mathrm{ml}$ of VEGF added to media. Sprouting was quantified after 5 days $(n=4,>30$ beads/sample). Sprouting increased with increasing concentrations of VEGF. Statistical significance of $P<$ 0.05 for all conditions, except where indicated by NS where $P>0.05$ relative to 10 and $50 \mathrm{ng} / \mathrm{ml}$ conditions. $c$ ) MEC sprouting was quantified in a chamber where VEGF was provided on 1 side of the fibrin gel and allowed to diffuse across, creating a gradient of VEGF. Number of sprouts per bead was counted in all beads within $1.0 \mathrm{~mm}$ of VEGF source after 5 days $(n=3$, $>50$ beads/sample). Level of sprouting decreased with increased distance away from VEGF source. Error bars $=$ SE. $*$ Indicates statistical significance from conditions marked by **, $P<0.05$. d) Angle of extension of a sprout from a bead (i.e., alignment of a sprout along a gradient) was measured according to the assigned axes in a nongradient control and in categories of increasing distance from the VEGF source: $0-0.2 \mathrm{~mm}, 0.2-0.4,0.4-0.6,0.6-0.8,0.8-1.0$, and $>1.0 \mu \mathrm{m}$. Sprouts were analyzed after 5 days $(n=3,>50$ beads/sample). Sprouts preferentially aligned along the gradient, with more sprouts closer to the VEGF source. MEC sprouting $(e)$ and alignment $(f)$ were quantified in a diffusion chamber with varying VEGF source concentrations: 10,100 , and $300 \mathrm{ng} / \mathrm{ml} . \mathrm{g}$ ) Dependence of sprouting on slope of gradient was determined in diffusion chamber sprouting assay using the midrange VEGF source concentration $(100 \mathrm{ng} / \mathrm{ml})$ that elicited a maximal sprouting response. Guidance of sprouting directionality occurs optimally with a gradient $>25 \mathrm{ng} / \mathrm{ml}$ per $\mathrm{mm}$.

levels of sprouting were observed from cells at high VEGF concentrations near the VEGF source in the chamber (Fig. 1c), as expected. Strikingly, quantification revealed that the proportion of sprouts aligned with the gradient was highest for cells close to the VEGF source and thus experiencing a combination of a high VEGF concentration and gradient (Fig. 1d). At distances farther from the VEGF source, sprouting was still observed, but the sprouts that formed showed no directional preference. Repeating this experiment for MECs in the presence of a high VEGF concentration without a gradient resulted in no preferential sprout alignment (Fig. 1d).
To further investigate VEGF control over sprout directionality, the sprouting assay was performed in the diffusion chamber under different source concentrations of VEGF (30, 100, and $300 \mathrm{ng} / \mathrm{ml})$. The resulting concentrations of VEGF within the fibrin gel were determined in parallel. Quantification of sprouting under these conditions revealed maximum sprouting at a VEGF concentration of $100 \mathrm{ng} / \mathrm{ml}$ (Fig. 1e). The presence of a VEGF gradient was critical to MEC sprout alignment, and cells were able to align along a VEGF gradient even at low concentrations. However, alignment began to decrease at VEGF concentrations $>250$ $\mathrm{ng} / \mathrm{ml}$ (Fig. $1 f$ ), possibly due to VEGF receptor satura- 
tion. Optimal sprout alignment occurred in the presence of a gradient $>25 \mathrm{ng} / \mathrm{ml}$ per $\mathrm{mm}$ (Fig. $1 \mathrm{~g}$ ).

A FRET technique (18) was next developed to quantify VEGF binding upon individual sprouting MECs, and to directly examine the relation between actual VEGF binding and the decision of individual cells to participate in sprout formation. FRET is widely used to examine nanoscale interactions of molecules (18) in 3-D but does not typically provide information regarding the specific numbers of interactions. In contrast, classic radiolabeled receptor binding assays $(9,10)$ are used to quantify ligand binding to cell receptors, but this technique is not amenable to real-time cell processes in 3-D culture. Parallel analysis of cells in 2-D culture, with both FRET measurements of VEGF binding and quantification of iodinated VEGF binding to cells, allowed calibration of the FRET signal to actual numbers of bound VEGF molecules (see Supplemental Fig. 2). The radiolabeled receptor binding assay demonstrated saturation between $40-100 \mathrm{ng} / \mathrm{ml}$ VEGF, with a receptor density of $2.3 \times 10^{5}$ receptors/cell (see Supplemental Fig. 2a). FRET was performed by labeling MECs with a lipophilic donor fluorescein dye, while VEGF was labeled with an acceptor rhodamine analog. The calibration curve allows one to calculate the number of VEGF molecules bound and taken up by sprouting MECs, based on the specific fluorescence intensity of cells in the assay (Fig. 2a). In general, both VEGF binding and sprouting increased with escalation of VEGF availability in the gel (Fig. 2b). The difference in VEGF binding between tip and nontip cells decreased as VEGF concentration in the media was increased (Fig. 2c).

A mathematical model of VEGF distribution in vivo was used to design a system capable of delivering VEGF in a spatial concentration gradient within the range where VEGF is able to both initiate and spatially control angiogenesis. Inputs to this model included the experimentally determined diffusion coefficient, half-life, and cellular uptake of VEGF (see Supplemental Fig. 3). This model led to a layered PLG scaffold design, in which the scaffold consisted of two layers with differen- tial VEGF loading in each layer $(1.5 \mu \mathrm{g}$ in layer 1 and 3 $\mu \mathrm{g}$ in layer 2). Incorporation of fluorescently labeled protein into scaffolds showed that the protein remained compartmentalized at the completion of the fabrication process (Fig. 3a). VEGF release was determined using radiolabeled VEGF, and the release kinetics varied based on the total loading of VEGF, resulting in different quantities of VEGF released from each layer of the scaffold (Fig. 3b). The predicted VEGF concentrations in tissue resulting from this delivery system fell within the desired active range (Fig. $3 c$ ), and appropriate orientation of the system in ischemic hindlimbs of mice could lead to a VEGF gradient that would drive vessels to extend in the desired direction. At steady state, a steep gradient of $40 \mathrm{ng} / \mathrm{ml}$ per $\mathrm{mm}$ existed along the tissue-scaffold interface, with a maximum tissue concentration of $120 \mathrm{ng} / \mathrm{ml}$ and a minimum concentration of $38 \mathrm{ng} / \mathrm{ml}$ (Fig. $3 c$ ). The gradient flattened with increasing distance away from the scaffold, but a gradient of VEGF in the tissue was maintained at steady state up to $1 \mathrm{~mm}$ away from the scaffold (Fig. 3c).

Layered scaffolds capable of delivering spatially controlled gradients of VEGF were implanted in a mouse model of hindlimb ischemia to assess their ability to drive angiogenesis and restore perfusion. The layered scaffolds were implanted such that the VEGF gradient extended perpendicularly across the ischemic limb, in the desired direction for new vessel extension. Controls included implantation of blank scaffolds (no VEGF), and scaffolds that had the same total quantity of VEGF evenly distributed throughout their volume (leading to similar VEGF concentrations in tissue, but little to no VEGF gradient in the desired direction). Spatial VEGF delivery led to extensive formation of new vessel networks in the ischemic limbs (Fig. 3d), while control conditions exhibited less extensive vessel networks (Fig. $3 e$ ), as indicated by micro-CT imaging of vascular casts. Analysis of vasculature revealed higher vessel densities in VEGF control and layered VEGF conditions than in the blank scaffold condition. In hindlimbs implanted with blank scaffolds, the sites of ligation were not fully
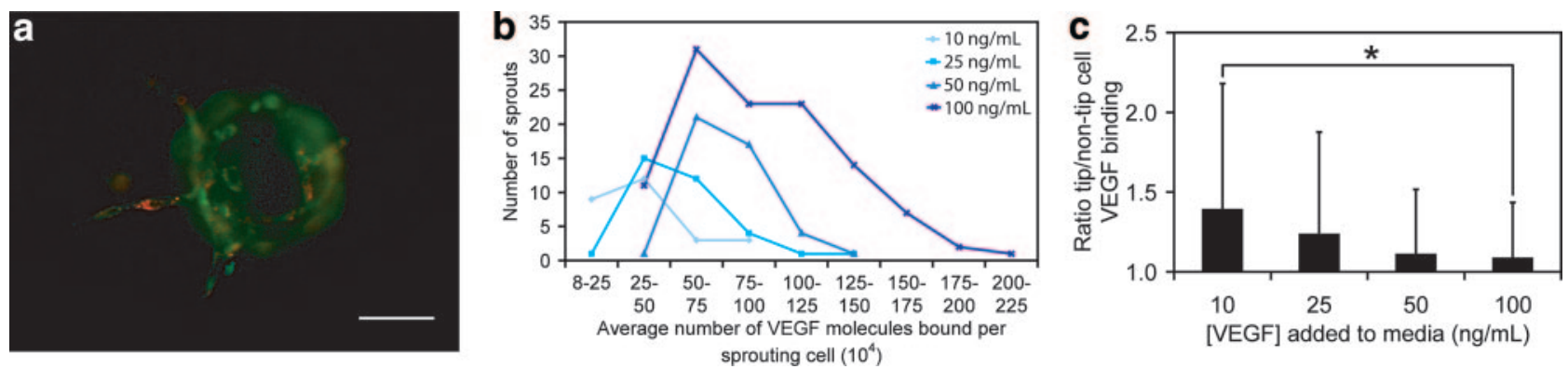

Figure 2. VEGF binding on sprouting MECs in vitro. a) Representative FRET image of labeled MECs (green) in a sprouting assay with labeled VEGF (red) shows energy transfer after excitation of donor fluorophore. Images of sprouts bound with VEGF were analyzed to quantify VEGF binding in individual sprouts via use of the calibration between FRET and iodinated VEGF binding. Scale bar $=50 \mu \mathrm{m}$. b) Histogram of VEGF bound/cell and the resultant extent of sprouting. Fluorescently labeled VEGF was added to media in a MEC sprouting assay $(n=4,>200$ beads/sample). Increasing concentrations of VEGF were used: 10,25 , 50 , and $100 \mathrm{ng} / \mathrm{ml}$. As VEGF availability increased, number of sprouts increased, and average VEGF bound to sprouting cells increased. c) VEGF binding specifically to tip cells $v s$. nontip MECs participating in sprouting was quantified. Overall, tip cells bound more VEGF than nontip cells, but this difference decreased with increasing VEGF availability. $* P<0.05$. 
a

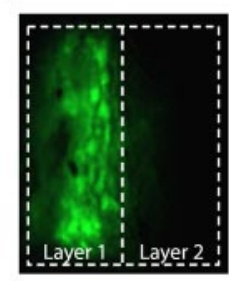

d

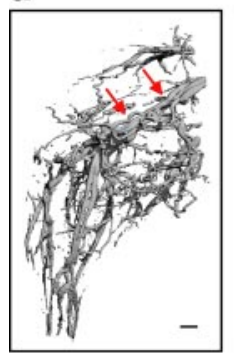

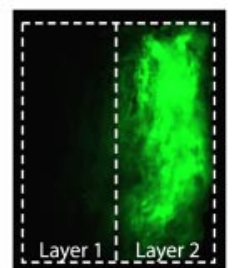

e

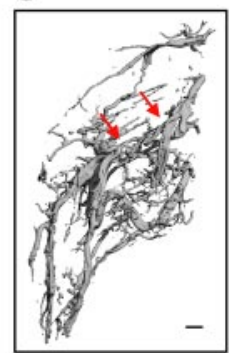

b

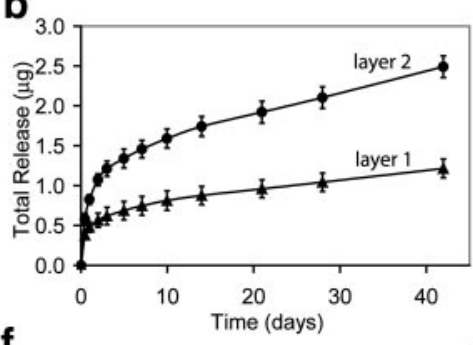

f

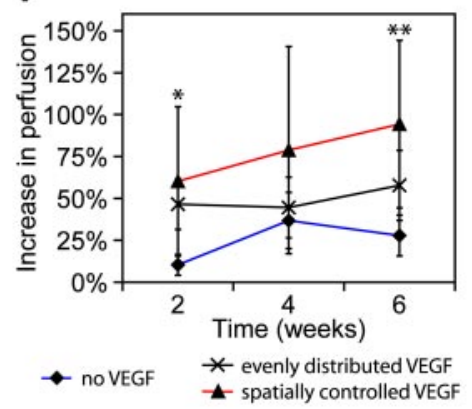

C

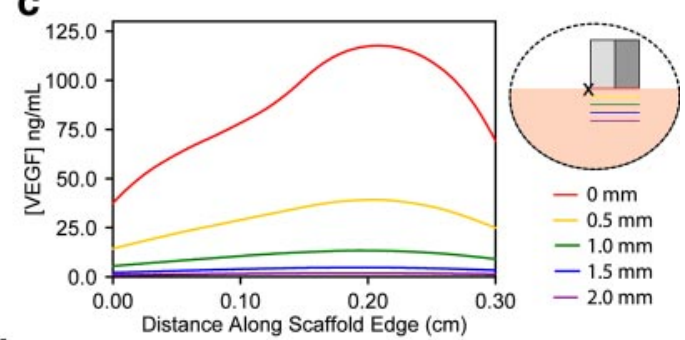

g

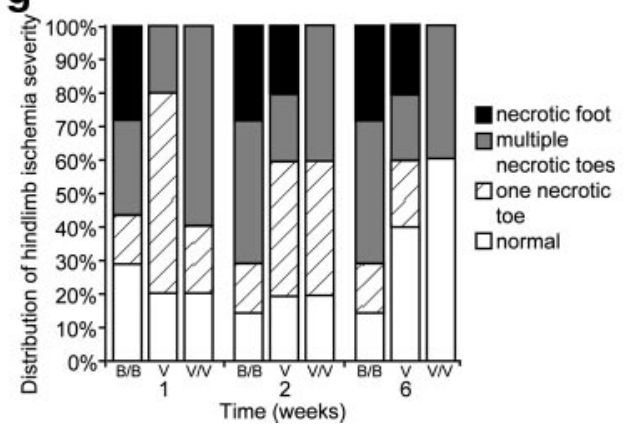

Figure 3. Spatially controlled VEGF delivery in vivo. a) Scaffolds with spatially compartmentalized VEGF were designed, based on the results of mathematical models of VEGF release needed to generate appropriate tissue concentrations and gradients. Fluorescently labeled protein was used to examine incorporation into individual compartments of layered scaffolds. After fabrication, loaded protein remained confined to each layer. Dotted lines indicate border of entire layered scaffold and separation between layers. $b$ ) VEGF release from each layer was determined using ${ }^{125}$ I-VEGF incorporated as a tracer in layer 1 or layer 2. Overall release profile is similar in each layer, with an initial burst of VEGF followed by a steady release. Quantity of VEGF released was proportional to total mass of VEGF incorporated in each layer (1.5 $\mu \mathrm{g}$ in layer 1 and $3 \mu \mathrm{g}$ in layer 2). c) Predicted VEGF distribution in ischemic hindlimbs, with scaffold orientation as indicated in insert. Scaffold placement was designed to create a VEGF gradient that extended away from the femoral artery/vein (X) into muscle tissue (pink), guiding new capillaries to orient down ischemic limb. VEGF concentrations in a layered scaffold were modeled and solved in COMSOL, and steady-state VEGF concentrations, predicted to be reached after $30 \mathrm{~min}$, are shown. Tissue VEGF concentrations across scaffold edge are plotted at distances away from edge: $0,0.5,1.0,1.5$, and $2.0 \mathrm{~mm}$. $d$, e) Micro-CT images of vascular casts show architecture of vascular network in ischemic hindlimbs. Layered VEGF scaffold implantation $(d)$ resulted in more normalized vasculature where the femoral artery and vein are completely bridged by a large vessel and the hypogastric vein is clearly visible without significant collateral arteriogenesis, while control scaffold (no VEGF) implantation $(e)$ did not result in complete bridging of the ligation sites (arrows) and collateral vessels were enlarged. Scale bar $=1 \mathrm{~mm} . f$ ) Recovery of hindlimb perfusion in animals that had undergone unilateral vessel ligation and scaffold implantation as monitored by LDPI $(n=5)$. Increase in perfusion over baseline after vessel ligation is plotted. Experimental conditions examined were control (no VEGF) scaffolds, scaffolds with $4.5 \mu \mathrm{g}$ VEGF evenly distributed, and spatially controlled VEGF delivery. Complete reperfusion was observed with spatially controlled presentation of VEGF. $* P<0.05$ for even and layered VEGF conditions compared to blank, $* * P<0.05$ for layered VEGF compared to blank, and $* * * P<0.05$ for layered VEGF compared to blank and even VEGF. $g$ ) Ischemic hindlimbs were examined to determine the severity of hindlimb ischemia postligation and scaffold implantation. Degree of hindlimb severity was categorized as normal compared to the unligated limb, or having one necrotic toe, multiple necrotic toes, or a necrotic foot. Hindlimb ischemia progressed through week 6 in animals implanted with blank control scaffolds (B/B). Animals with evenly distributed VEGF (V) had lower levels of ischemic severity compared to control, but necrosis continued to progress through week 6 . Spatially regulated VEGF presentation $(\mathrm{V} / \mathrm{V})$ reduced limb necrosis at week 1 , and improvements in hindlimb ischemia occurred over time, with a resultant prevention of severe necrosis.

bridged and enlargement of collateral vessels (anterior tibial artery and vein, lateral marginal artery and vein) occurred, indicating occluded blood flow in nearby arteries. In vascular casts of hindlimbs with layered VEGF scaffold implantation, the sites of ligation were bridged by a large conductance vessel, re-establishing the femoral artery. Additionally, there were fewer enlarged vessels around the site of the spatial VEGF delivery, and the architecture of major arteries and veins (hypogastric, great saphenous, anterior tibial, lateral marginal) was clearly visible with close resemblance to that of normal hindlimbs. The vascular network in the area of layered scaffold implantation showed vascular organization corresponding to the delivered VEGF gradient, where vessel density increased away from the sites of ligation.

Importantly, spatial VEGF delivery resulted in rapid restoration of hindlimb blood flow to normal levels by week 2 (Fig. $3 f$ ). Control animals implanted with a blank scaffold had a small increase in perfusion to $46 \pm$ $5 \%$ of the normal level, whereas VEGF delivery without spatial control led to perfusion levels of $63 \pm 16 \%$ by this time. The varying ability of the experimental interventions to increase hindlimb blood flow was reflected in observed levels of hindlimb ischemia severity (Fig. $3 g$ ). Severe necrosis occurred with blank and uniform VEGF delivery, and the integrity of ischemic hindlimbs declined over time in these conditions. The 
rapid restoration of blood flow to ischemic hindlimbs achieved with spatial VEGF delivery had a significant protective effect against severe hindlimb necrosis and improved hindlimb integrity over time (Fig. $3 g$ ). Since blood flow largely returned to normal by week 2 , toes that initially appeared necrotic in this group also returned to normal color by week 6 .

\section{DISCUSSION}

The results from these in vitro studies further elucidate and quantify the role of $\operatorname{VEGF}(165)$ in controlling the biological process of angiogenesis. Previous in vitro work has shown that VEGF not only enhances endothelial cell survival, proliferation, and migration $(19,20)$ but that VEGF is also a potent chemoattractant (21) and provides directionality to sprouting endothelial cells (22). In addition to supporting previous findings, data from these studies show VEGF binding in longer term cultures was higher than levels noted at saturation in 2-D culture (Fig. 2b; also see Supplemental Fig. 3c), suggesting that either VEGF exposure or 3-D culture led to increased receptors on MECs. Therefore, quantification of VEGF binding in an in vitro 3-D model of sprouting angiogenesis is essential to understanding VEGF control of this process.

Importantly, the data from 3-D culture indicate VEGF control over sprouting and its direction results from heterogeneity in the ability of MECs to bind and respond to VEGF. Although no sprouting was noted when $<8 \times 10^{4}$ molecules VEGF were bound per cell (Fig. 2b), there did not appear to be one threshold of binding where all cells sprouted. This finding suggests that two thresholds may guard MEC activation and sprout initiation. Quantification of VEGF binding also revealed heterogeneity in VEGF binding among sprouting cells, as the cells leading the sprout (tip cells) had a statistically significantly increase in VEGF binding as compared to the cells in the body of the sprout (nontip cells). This finding supports previous in vivo studies showing that vascular sprouts are composed of distinct populations of nontip cells led by migrating tip cells (23). Sprout alignment along a VEGF gradient may be facilitated by this ability of tip cells to bind higher numbers of VEGF molecules and thus become activated at lower VEGF concentrations and detect subtle differences in VEGF concentration as present in VEGF gradients. As VEGF concentration in the media increased, the difference between VEGF binding on tip and nontip cells decreased (Fig. 2c), presumably due to cell receptors becoming saturated, and this contributed to the loss of sprout alignment at high VEGF concentrations.

The conclusions drawn from the data suggest there is a both a minimum concentration and gradient of VEGF required for control over sprouting and its direction. Low VEGF concentrations may be insufficient to activate MECs toward sprout formation, while high VEGF concentrations may saturate cell receptors and disrupt cell guidance. These studies indicate that control over angiogenesis is likely most effective when VEGF is present in a gradient, with a maximum concentration near $100 \mathrm{ng} / \mathrm{ml}$ for optimal initiation of sprouting and control over directionality.

An effective in vivo growth factor delivery system was created based on this quantitative understanding of the role of VEGF in controlling angiogenesis in vitro. SCID mice that had undergone unilateral external iliac and femoral artery ligation were used as a severe model of hindlimb ischemia because unlike normal mice, these animals are not able to fully recover hindlimb perfusion without intervention (24). The results from implantation of devices with uniform VEGF distribution in ischemic hindlimbs of SCID mice were consistent with past studies $(16,24)$ where hindlimb blood flow was enhanced above control, but did not completely restore perfusion to normal levels. Interestingly, hindlimbs implanted with devices designed to deliver a spatial VEGF gradient based on in vitro modeling showed accelerated recovery from hindlimb ischemia (Fig. $3 f$ ), and this result supports the importance of VEGF tissue gradients in addition to VEGF concentrations in forming functional vessel networks.

The in vivo results highlight the importance of accelerated recovery from hindlimb ischemia to prevent the incidence of severe necrosis (Fig. $3 g$ ). These results also indicate that spatial VEGF delivery may have led to the formation of more normalized vascular networks, allowing accelerated restoration of perfusion. The amplified reperfusion described in this study was likely due to both the sustained delivery of VEGF and the spatially controlled presence of VEGF. Spatially controlled VEGF delivery may direct the initial formation of a more organized vascular network, reducing the need for significant remodeling and enabling efficient network formation.

In these studies, we have demonstrated that a quantitative understanding of the growth factor driven process of angiogenesis can be used to rationally design delivery vehicles, leading to improved therapeutic outcomes. This integration of quantitative biological measurements, mathematical modeling, and device design has great potential utility not only for therapeutic angiogenesis but also for other tissue regeneration strategies using growth factor delivery, and highlights the importance of interdisciplinary approaches in designing molecular therapies.

We gratefully acknowledge financial support from the National Institutes of Health (R01 HL-069957) and the Biological Resources Branch of the National Cancer Institute for providing VEGF for our studies.

\section{REFERENCES}

1. Rosinberg, A., Khan, T. A., Sellke, F. W., and Laham, R. J. (2004) Therapeutic angiogenesis for myocardial ischemia. Expert. Rev. Cardiovasc. Ther. 2, 271-283

2. Lazarous, D. F., Shou, M., Scheinowitz, M., Hodge, E., Thirumurti, V., Kitsiou, A. N., Stiber, J. A., Lobo, A. D., Hunsberger, 
S., Guetta, E., et al. (1996) Comparative effects of basic fibroblast growth factor and vascular endothelial growth factor on coronary collateral development and the arterial response to injury. Circulation 94, 1074-1082

3. Chen, R. R., and Mooney, D. J. (2003) Polymeric growth factor delivery strategies for tissue engineering. Pharm. Res. 20, 11031112

4. Conway, E. M., Collen, D., and Carmeliet, P. (2001) Molecular mechanisms of blood vessel growth. Cardiovasc. Res. 49, 507-521

5. Khurana, R., Simons, M., Martin, J. F., and Zachary, I. C. (2005) Role of angiogenesis in cardiovascular disease: a critical appraisal. Circulation 112, 1813-1824

6. Nehls, V., and Drenckhahn, D. (1995) A novel, microcarrierbased in vitro assay for rapid and reliable quantification of three-dimensional cell migration and angiogenesis. Microvasc. Res. 50, 311-322

7. Dietrich, F., and Lelkes, P. I. (2006) Fine-tuning of a threedimensional microcarrier-based angiogenesis assay for the analysis of endothelial-mesenchymal cell co-cultures in fibrin and collagen gels. Angiogenesis 9, 111-125

8. Russ, J. (2002) The Image Processing Handbook, CRC Press, Cleveland, OH, USA

9. Davenport, A. (2005) Receptor Binding Techniques, Humana Press, Totowa, NJ, USA

10. Limbird, L. E. (1996) Cell Surface Receptors: a Short Course on Theory and Methods, Kluwer Academic Publishers, Boston, MA, USA

11. Soker, S., Fidder, H., Neufeld, G., and Klagsbrun, M. (1996) Characterization of novel vascular endothelial growth factor (VEGF) receptors on tumor cells that bind VEGF165 via its exon 7-encoded domain. J. Biol. Chem. 271, 5761-5767

12. Vaisman, N., Gospodarowicz, D., and Neufeld, G. (1990) Characterization of the receptors for vascular endothelial growth factor. J. Biol. Chem. 265, 19461-19466

13. Richardson, T. P., Peters, M. C., Ennett, A. B., and Mooney, D. J. (2001) Polymeric system for dual growth factor delivery. Nat. Biotechnol. 19, 1029-1034

14. Harris, L. D., Kim, B. S., and Mooney, D. J. (1998) Open pore biodegradable matrices formed with gas foaming. J. Biomed. Mater. Res. 42, 396-402
15. Peters, M. C., Polverini, P. J., and Mooney, D. J. (2002) Engineering vascular networks in porous polymer matrices. J. Biomed. Mater. Res. 60, 668-678

16. Sun, Q., Chen, R. R., Shen, Y., Mooney, D. J., Rajagopalan, S., and Grossman, P. M. (2005) Sustained vascular endothelia growth factor delivery enhances angiogenesis and perfusion in ischemic hind limb. Pharm. Res. 22, 1110-1116

17. Nakatsu, M. N., Sainson, R. C., Perez-del-Pulgar, S., Aoto, J. N., Aitkenhead, M., Taylor, K. L., Carpenter, P. M., and Hughes, C. C. (2003) VEGF(121) and VEGF(165) regulate blood vessel diameter through vascular endothelial growth factor receptor 2 in an in vitro angiogenesis model. Lab. Invest. 83, 1873-1885

18. Sekar, R. B., and Periasamy, A. (2003) Fluorescence resonance energy transfer (FRET) microscopy imaging of live cell protein localizations. J. Cell Biol. 160, 629-633

19. Hutchings, H., Ortega, N., and Plouet, J. (2003) Extracellular matrix-bound vascular endothelial growth factor promotes endothelial cell adhesion, migration, and survival through integrin ligation. FASEB J. 17, 1520-1522

20. Ruhrberg, C. (2003) Growing and shaping the vascular tree: multiple roles for VEGF. Bioessays 25, 1052-1060

21. Coultas, L., Chawengsaksophak, K., and Rossant, J. (2005) Endothelial cells and VEGF in vascular development. Nature 438, 937-945

22. Eichmann, A., Le Noble, F., Autiero, M., and Carmeliet, P. (2005) Guidance of vascular and neural network formation. Curr. Opin. Neurobiol. 15, 108-115

23. Gerhardt, H., Golding, M., Fruttiger, M., Ruhrberg, C., Lundkvist, A., Abramsson, A., Jeltsch, M., Mitchell, C., Alitalo, K. Shima, D., and Betsholtz, C. (2003) VEGF guides angiogenic sprouting utilizing endothelial tip cell filopodia. J. Cell Biol. 161, 1163-1177

24. Chen, R. R., and Mooney, D. J. (2006) Host immune competence and local ischemia affects the functionality of engineered vasculature. Microcirculation 14, 77-88.

Received for publication February 19, 2007. Accepted for publication June 7, 2007. 